\title{
Phylogeography of the threatened crayfish (genus Austropotamobius) in Italy: implications for its taxonomy and conservation
}

\author{
S Fratini ${ }^{1}$, S Zaccara ${ }^{2}$, S Barbaresi ${ }^{1}$, F Grandjean ${ }^{3}$, C Souty-Grosset ${ }^{3}$, G Crosa ${ }^{2}$ and F Gherardi ${ }^{1}$ \\ ${ }^{1}$ Dipartimento di Biologia Animale e Genetica 'L. Pardi', Università di Firenze, v. Romana 17, 50125 Firenze, Italy; ${ }^{2}$ Dipartimento di \\ Biologia Strutturale e Funzionale, Università dell'Insubria, via Dunant 3, 21100 Varese, Italy; ${ }^{3}$ Laboratoire de Génétique et Biologie des \\ Populations de Crustacés, UMR 6556, Université de Poitiers, 40 avenue du Recteur Pineau, F-86022 Poitiers cedex, France
}

A nucleotide sequence analysis of a portion of the mitochondrial large ribosomal subunit was performed to define the phylogeography of the threatened crayfish Austropotamobius (Decapoda; Astacidae) in Italy. We collected 61 specimens from 31 localities across the Italian peninsula. For the phylogenetic inference, we combined the 61 Austropotamobius spp sequences obtained from this study with 18 sequences deposited in GenBank and corresponding to Italian, French, Irish, Swiss, and Slovenian locations. Among the analysed sequences, 34 distinct haplotypes were detected. Our results confirmed the presence of both $A$. pallipes and $A$. italicus in the Italian peninsula and the existence within the latter species of a strong intraspecific genetic variation, due to the occurrence of four subspecies with a well-defined geographic distribution. From a conservation viewpoint, Italy, with its high haplotype variability, may be considered a 'hot spot' for the genetic diversity of the European native crayfish Austropotamobius. We suggest that re-introduction programs should be conducted with extreme caution in Italy, since not only the two Austropotamobius species but also the four $A$. italicus subspecies are genetically and taxonomically separate units and require independent conservation plans.

Heredity (2005) 94, 108-118. doi:10.1038/sj.hdy.6800581

Published online 13 October 2004

Keywords: Austropotamobius; freshwater crayfish; phylogeny; phylogeography; mitochondrial DNA; conservation

\section{Introduction}

The assessment of the phylogeography of a species and the identification of genetically divergent areas is a fundamental step for the success of any conservation effort. Genetic variability is widely recognized as a component of natural biodiversity, several national and international conventions and laws claiming the necessity of its preservation and protection (Soulé and Mills, 1992; Primack, 2000). Phylogeography is a powerful tool for inferring the processes that affect the genetic composition of species or species groups; it can be helpful in elucidating historical events, such as habitat fragmentation and range expansion, which have influenced the population structure of a species or have caused speciation. Finally, thanks to the adoption of biomolecular techniques in the field of conservation biology, phylogeography studies have permitted the identification of Evolutionary Significant Units (ESUs), that is, those sets of populations reciprocally monophyletic for mitochondrial DNA (mtDNA) that show significant divergences in allele frequencies at nuclear loci (Moritz, 1994).

Due to the increasing loss and degradation of freshwater habitats throughout the world, the conservation of

Correspondence: S Fratini, Dipartimento di Biologia Animale e Genetica 'L. Pardi', Università di Firenze, v. Romana 17, 50125, Firenze, Italy. E-mail:sarafratini@unifi.it

Received 24 October 2003; accepted 27 June 2004; published online 13 October 2004 freshwater species is becoming more and more urgent (Erwin, 1991). A paradigmatic case is the European white-clawed crayfish Austropotamobius pallipes (Astacidae; Decapoda), a species distributed from the United Kingdom to Italy and Yugoslavia. In the last few decades, the survival of this species has been also threatened by the introduction into Europe of exotic crayfish, acting as strong competitors for resources and vectors of the crayfish plague. At present, the species is considered vulnerable by the IUCN (Baillie and Groombridge, 1996) and is protected by the 'Council directive 92/43/ECC'.

The lack of an adequate taxonomic classification and difficulty in using distinct morphological characters for the assessment of different taxa are two reasons that often make particularly difficult the effective management of at-risk species. Systematic uncertainties can, in fact, cause the application of erroneous conservation procedures, with dramatic consequences for the species survival (Frankham et al, 2002). A. pallipes is a key example in this scenario: in the last five decades, in order to explain the high intraspecific variability recorded within its distribution area (Bott, 1950; Karaman, 1962; Brodsky, 1983; Starobogatov, 1995), this species has gone through several taxonomic revisions that proposed different criteria on how to classify the various morphologically and genetically distinct groups so far identified. The matter was made even more complex due to the unclear and even misleading morphological differences found in this taxon. The current position (Grandjean et al, 2000, 2002a, b), based on $16 S$ rRNA and supported by 
morphological (Bott, 1950; Karaman, 1962; Brodsky, 1983; Grandjean et al, 1998) and allozymatic studies (Santucci et al, 1997) is that A. pallipes is a species complex with a strong genetic structure, both at inter- and intraspecific levels. The complex is formed of two genetically well distinct species, $A$. pallipes and $A$. italicus, with three subspecies: (1) A. i. italicus distributed in Italy, South Switzerland, and Spain; (2) A. i. carsicus distributed in the Balkans, and (3) A. i. carinthiacus distributed in Austria and Switzerland. The presence of an endemic subspecies in Spain, A. i. lusitanicus, has been accepted since Bott (1950), but Santucci et al (1997) and Grandjean et al (2000, $2002 b$ ) showed the absence of genetic differentiation between this and the Italian subspecies (A. i. italicus). The existence of an endemic species in Southern Switzerland (A. berndhauseri), first proposed by Bott (1972), has been excluded by Grandjean et al (2002a), who demonstrated that this corresponds to $A$. $i$. carinthiacus.

The presence in Italy of both $A$. pallipes and $A$. italicus has been proven by several studies (Lörtscher et al, 1997; Nascetti et al, 1997; Santucci et al, 1997; Grandjean et al, 2000; Largiadèr et al, 2000), showing that the former is confined to North-Western Italy and the latter is distributed across the entire peninsula. Santucci et al (1997) and Nascetti et al (1997) found that the two species overlap in the Ligurian Apennine without, however, showing hybridization events. These authors also revealed a strong genetic structure within A. italicus in Italy and defined the following main population clusters, partially related to their geographic distribution: (1) North-Central Apennines; (2) Latium, Abruzzi, and Southern Italy; and (3) North-Eastern Italy. Such a genetic structure specific to $A$. italicus suggests the existence of distinct $A$. italicus subspecies in Italy, but no subsequent studies have been conducted to assess this hypothesis.

The aim of this study was to investigate the phylogeography of the white-clawed crayfish across the Italian peninsula. From a conservation viewpoint, knowledge of the geographic distribution of Austropotamobius genetic variability in Italy may contribute to defining management programs for this threatened species. For this purpose, we used the sequence of the mitochondrial ribosomal large subunit (mtDNA rRNA 16S), in the light of (a) its higher resolution power with respect to allozymes (Crandall, 1996), (b) its potential in detecting genetic differentiation in crustaceans, and especially in crayfish (Crandall, 1996), and (c) because the taxonomy of Austropotamobius is currently based on this genetic marker (Grandjean et al, 2000, 2002a, b). This last point allowed us to discuss our results in a systematic perspective.

\section{Materials and methods}

\section{Crayfish sampling}

A total of 61 crayfish were collected by hand from 31 sites across the range of $A$. spp in Italy (Figure 1 and Table 1). A chela or a pereopod was taken from each of the 61 individuals and was immediately put in a vial containing absolute ethanol. The specimens were preserved in a glass container with $70 \%$ ethanol and then catalogued at the museums of the Universities of Florence (Italy) and Poitiers (France), for future morphological studies.

\section{DNA extraction and amplification}

Total genomic DNA was extracted from muscle tissues by multiple extraction methods (Qiagen tissue DNA extraction kit and phenol-chloroform-isoamyl method; Kocher et al, 1989). The DNAs were eluted in a buffer supplied by Qiagen or in water, and stored at $+4^{\circ} \mathrm{C}$ for routine use and at $-20^{\circ} \mathrm{C}$ for long-term preservation. Selective amplification of a rDNA 16 S portion, about 550 base pairs (bp) long, was carried out by polymerase chain reaction (PCR) using primer 1471 (5'-CCT GTTTANCAAAACAT-3') and primer 1472 (5'-AGATAG AAACCAACCTGG-3') from Crandall and Fitzpatrick (1996), with the following PCR conditions: 45 cycles for $60 \mathrm{~s}$ at $95^{\circ} \mathrm{C}$ for denaturation, $60 \mathrm{~s}$ at $45^{\circ} \mathrm{C}$ for annealing, $60 \mathrm{~s}$ at $72^{\circ} \mathrm{C}$ for extension, preceded by $3 \mathrm{~min}$ of initial denaturation at $95^{\circ} \mathrm{C}$ and followed by $5 \mathrm{~min}$ of final extension at $72^{\circ} \mathrm{C}$; and primer $16 \mathrm{Sar}\left(5^{\prime}\right.$-CGCCTGTTTAT CAAAAACAT- $3^{\prime}$ ) and primer 16Sbr (5'-CCGGTCTGAA CTCAGATCACGT-3') from Palumbi et al (1991) with the following PCR conditions: 40 cycles for $30 \mathrm{~s}$ at $94^{\circ} \mathrm{C}$ for denaturation, $30 \mathrm{~s}$ at $47^{\circ} \mathrm{C}$ for annealing, $45 \mathrm{~s}$ at $72^{\circ} \mathrm{C}$ for extension, preceded by $5 \mathrm{~min}$ of initial denaturation at $94^{\circ} \mathrm{C}$ and followed by $10 \mathrm{~min}$ of final extension at $72^{\circ} \mathrm{C}$. The two sets of primers work equally well in both the Austropotamobius species.

Successful PCR products were purified by the ExoSAP-IT buffer $\left(\mathrm{USB}^{\circledR}\right.$ ) or a GeneClean II kit (Bio 101), and then sequenced using the Big Dye Terminator method (PE Applied Byosystem) on an ABI 377 automated sequencer. For most samples, the forward and reverse sequences were obtained. Sequence data were submitted to GenBank (accession numbers in Table 1).

\section{Sequence analysis}

Sequences were aligned by eye using the software ESEE Version 3.2 (based on Cabot and Beckenbach, 1989). The data matrix included the 61 sequences examined in this study and 18 sequences of Austropotamobius spp, obtained by Grandjean et al (2000) and Largiadèr et al (2000), and deposited in GenBank (Table 1). These sequences from GenBank correspond both to Italian sites (Figure 1 and Table 1) and to French, Irish, Swiss, and Slovenian locations (Table 1 ). A sequence of $A$. torrentium from GenBank (AF237599; Grandjean et al, 2000) was also included as outgroup.

Phylogenetic inference was performed using the neighbor-joining (NJ), the maximum likelihood (ML), and the maximum parsimony (MP) analysis using PAUP* (Swofford, 1998). The optimal model of nucleotide evolution for ML and NJ analyses was determined by hierarchical likelihood ratio tests using the software WINMODELTEST $4 \mathrm{~b}$, which is implemented in the PAUP* program package (Swofford, 1998). This approach consists in successive pairwise comparisons of alternative substitution models in a hierarchical hypothesis-testing framework (Posada and Crandall, 1998).

ML and MP analyses were performed using a heuristic search, based on branch swapping with tree bisectionreconnection. The ML starting tree was obtained via stepwise addition and replicated 10 times, with each replicate starting with a random input order of sequences. For $\mathrm{MP}$, the ratio of transitions versus transversions $(s / v)$ was weighted at 1:5, as resulted from 


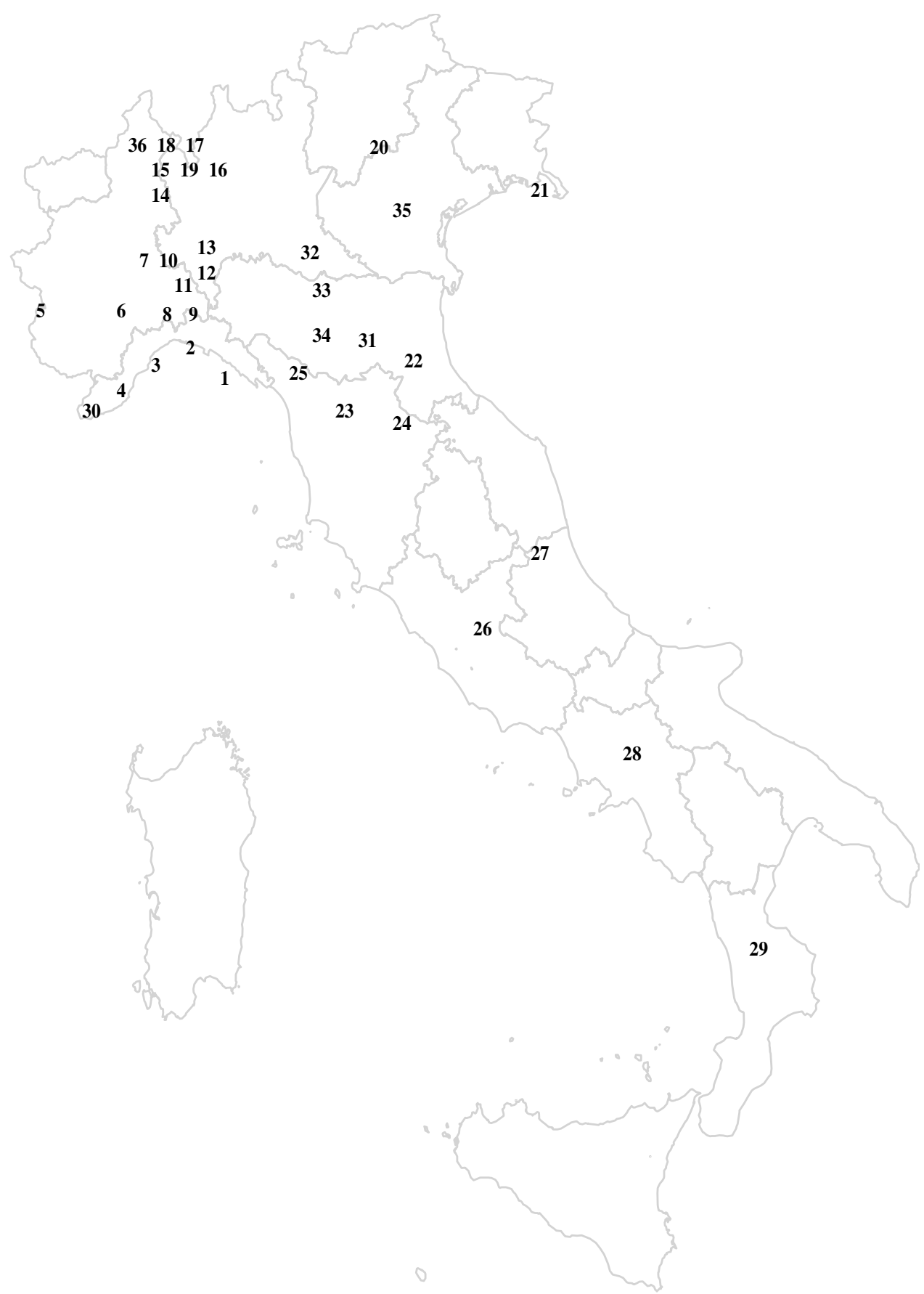

Figure 1 Distribution map of Italian Austropotamobius spp populations analysed in this study. Details for each population are reported in Table 1. No. 19 indicates three populations along the same water body.

the likelihood ratio test procedure, and gaps were excluded from the analysis.

For all methods, confidence values for the proposed groups within the inferred trees were calculated with the bootstrap method (Felsenstein, 1985).

\section{Results}

The sequence alignment consisted of $486 \mathrm{bp}$, primer regions excluded. We found 102 variable sites, 37 of which were parsimony informative, and 13 gaps.
Among all Austropotamobius sequences, 34 distinct haplotypes were detected (Table 1). The transitions to transversions $(s / v)$ ratio averaged 4.86 . The GC content ranged from 30.0 to $32.8 \%$; the recorded AT bias found in all the sequences is in agreement with that described for the arthropod mitochondrial genome (Clary and Wolstenholme, 1985). Table 2 reports the sequence divergences among the different haplotypes.

Applying the likelihood ratio tests procedure, the selected model of DNA substitution was the HKY85 model (Hasegawa et al, 1985) with unequal substitution rate and with a gamma distribution shape parameter 
Table 1 Italian (1-36) and not Italian (37-47) Austropotamobius spp populations analysed in this study

\begin{tabular}{|c|c|c|c|c|c|c|}
\hline \# & Water body & $\begin{array}{l}\text { Hydrographic } \\
\text { drainage }\end{array}$ & Country & $\begin{array}{l}\text { Sample } \\
\text { size }\end{array}$ & Haplotype (n) & $\begin{array}{l}\text { GenBank accession number, original source and } \\
\text { taxonomic reference }\end{array}$ \\
\hline 1 & Gottero & Magra & Italy & 2 & A3 (2) & AY611185 (present paper): A. i. carinthiacus \\
\hline 2 & Arvigo & Bisagno & Italy & 1 & A22 (1) & AY611202 (present paper): A. pallipes \\
\hline 3 & Nenno & Po & Italy & 1 & A23 (1) & AY611203 (present paper): A. pallipes \\
\hline 4 & Montenotte & Po & Italy & 1 & A24 (1) & AY611204 (present paper): A. pallipes \\
\hline 5 & Varaita & Po & Italy & 2 & A5 (2) & AY611201 (present paper): A. pallipes \\
\hline 6 & Visone & Po & Italy & 3 & A13 (3) & AY611192 (present paper): A. i. merdionalis \\
\hline 7 & Tanaro & Po & Italy & 3 & A1 (3) & AY611183 (present paper): A. i. carinthiacus \\
\hline 8 & Lemme & Po & Italy & 2 & A5 (2) & AY611201 (present paper): A. pallipes \\
\hline 9 & Borbera-Lagoscuro & Po & Italy & 4 & $\begin{array}{l}\text { A3 (1); A10 (1); } \\
\text { A11 (1); A12 (1) }\end{array}$ & AY611185 (present paper): A. i. carinthiacus \\
\hline 10 & Scrivia & Po & Italy & 2 & A1 (2) & AY611183 (present paper): A. i. carinthiacus \\
\hline 11 & Predrasso & Po & Italy & 2 & A1 (2) & AY611183 (present paper): A. i. carinthiacus \\
\hline 12 & Lazzuola & Po & Italy & 3 & A1 (3) & AY611183 (present paper): A. i. carinthiacus \\
\hline 13 & Schizzola & Po & Italy & 2 & A1 (2) & AY611183 (present paper): A. i. carinthiacus \\
\hline 14 & Ticino & Po & Italy & 3 & A3 (3) & AY611185 (present paper): A. i. carinthiacus \\
\hline 15 & Clivio & Po & Italy & 2 & A1 (1); A3 (1) & AY611183-85 (present paper): A. i. carinthiacus \\
\hline 16 & Lambro & Po & Italy & 2 & A1 (1); A3 (1) & AY611183-85 (present paper): A. i. carinthiacus \\
\hline 17 & Lambro & Po & Italy & 1 & A15 (1) & AY611195 (present paper): A. i. carsicus \\
\hline 18 & Lambro & Po & Italy & 2 & A2 (2) & AY611184 (present paper): A. i. carinthiacus \\
\hline 19 & Lambro & Po & Italy & 1 & A2 (1) & AY611184 (present paper): A. i. carinthiacus \\
\hline 19 & Lambro & Po & Italy & 2 & A16 (1); A17 (1) & AY611196-97 (present paper): A. i. carsicus \\
\hline 19 & Lambro & Po & Italy & 2 & A1 (1); A3 (1) & AY611183-85 (present paper): A. i. carinthiacus \\
\hline 20 & Lake Caldonazzo & Brenta & Italy & 1 & A18 (1) & AY611198 (present paper): A. i. carsicus \\
\hline 21 & Rosandra & Rosandra & Italy & 2 & A4 (2) & AY611186 (present paper): A. i. carsicus \\
\hline 22 & Lama & Bidente-Ronco & Italy & 2 & A8 (2) & AY611189 (present paper): A. i. italicus \\
\hline 23 & Farfereta & Arno & Italy & 2 & A6 (1); A7 (1) & AY611187-88 (present paper): A. i. italicus \\
\hline 24 & Staggia & Arno & Italy & 2 & A8 (2) & AY611189 (present paper): A. i. italicus \\
\hline 25 & Collegnago & Magra & Italy & 2 & A1 (2) & AY611183 (present paper): A. i. carinthiacus \\
\hline 26 & Duranna & Tevere & Italy & 2 & A9 (2) & AY611190 (present paper): A. i. merdionalis \\
\hline 27 & Nera & Tevere & Italy & 1 & A14 (1) & AY611193 (present paper): A. i. merdionalis \\
\hline 28 & S. Antuono & Sele & Italy & 2 & A13 (1); A14 (1) & AY611192-93 (present paper): A. i. merdionalis \\
\hline 29 & Coscile & Crati & Italy & 2 & A13 (1) & AY611192 (present paper): A. i. merdionalis \\
\hline 30 & Oxentina & Argentina & Italy & / & A21 & AF237597 (Grandjean et al, 2000): A. pallipes \\
\hline 31 & Samoggia & Reno & Italy & / & A19; A20 & AF237590-602 (Grandjean et al, 2000): A. i. italicus \\
\hline 32 & Taro & Po & Italy & / & A1 & AJ242706 (Largiader et al, 2000): A. i. carinthiacus \\
\hline 33 & Modolena & Po & Italy & / & A3 & AJ242705 (Largiader et al, 2000): A. i. carinthiacus \\
\hline 34 & Lake Botasso & Po & Italy & / & A1 & AJ242706 (Largiader et al, 2000): A. i. carinthiacus \\
\hline 35 & Monti Berici & Adige & Italy & / & V1; V2 & AJ242710-11 (Largiader et al, 2000): A. i. carsicus \\
\hline 36 & Lanza & Po & Italy & / & A1 & AJ242706 (Largiader et al, 2000): A. i. carinthiacus \\
\hline 37 & Rizana & Adriatic & Slovenia & / & A28 & AF237593 (Grandjean et al, 2000): A. i. carsicus \\
\hline 38 & Artix & Ariege & France & / & A25 & AF237610 (Grandjean et al, 2000): A. pallipes \\
\hline 39 & Val Renard & Orne & France & / & A27 & AF237595 (Grandjean et al, 2000): A. pallipes \\
\hline 40 & * & * & Ireland & / & A26 & AF237594 (Grandjean et al, 2000): A. pallipes \\
\hline 41 & * & Rhine & Switzerland & / & $\mathrm{N} 2$ & AJ242701 (Largiader et al, 2000): A. pallipes \\
\hline 42 & * & Rhine & Switzerland & / & N3 & AJ242702 (Largiader et al, 2000): A. pallipes \\
\hline 43 & * & Rhone & Switzerland & / & SW1 & AJ242708 (Largiader et al, 2000): A. i. carinthiacus \\
\hline 44 & * & Rhone & Switzerland & / & SW2 & AJ242709 (Largiader et al, 2000): A. i. carinthiacus \\
\hline 45 & * & Rhine/Rhone & Switzerland & / & A26 & AJ242703 (Largiader et al, 2000): A. pallipes \\
\hline 46 & * & Po & Switzerland & / & A1 & AJ242706 (Largiader et al, 2000): A. i. carinthiacus \\
\hline 47 & * & Po & Switzerland & / & $\mathrm{A} 3$ & AJ242705 (Largiader et al, 2000): A. i. carinthiacus \\
\hline
\end{tabular}

For each site, data are reported of: water body and its hydrographic drainage; country; sample size; mitochondrial 16S haplotypes (in parentheses, the number of individuals for each haplotype). Number 19 indicates three populations along the same water body. Numbers 1-29 correspond to the populations sampled for the present study; 30-47 to sequences downloaded from GeneBank (their accession number, original source, and taxonomic reference are indicated); 40-47 to haplotypes from more than one water body ${ }^{*}$ ).

equal to 0.2251 . This model was used to apply the $\mathrm{NJ}$ and ML analysis methods.

The MP method yielded one most parsimonious tree of length $82(\mathrm{CI}=0.87, \mathrm{RI}=0.95)$. The $-\mathrm{Ln}$ likelihood of the ML tree is 1092.8. Overall, all phylogenetic analyses resulted in a very congruent topology and the differences did not affect the general definition of clades and subclades (Figure 2).

The phylogenetic inference supports the separation of the haplotypes into two major clades (A and $\mathrm{B}$ ) corresponding to A. italicus and A. pallipes groups. Out of the 102 variable sites, six sites discriminate the two major groups (Table 3). The average genetic variation (calculated as $p$-distance $=$ number of substitutions/total number of nucleotides examined expressed in percentage) within A. pallipes and A. italicus clades is $0.34 \pm 0.16$ and $2.0 \pm 0.04 \%$, respectively; the average between the two species is $3.5 \pm 0.73 \%$. The sequence divergence ranges (in percentages) are: 0-3.6 between $A$. italicus haplotypes; $0-1.0$ between $A$. pallipes haplotypes; $2.6-4.3$ between $A$. pallipes and $A$. italicus haplotypes; $7.6-8.1$ between $A$. torrentium and $A$. pallipes 
Table 2 Pairwise sequence divergence (adjusted for missing data, calculated as $p$-distance $=$ number of substitutions/total number of nucleotides examined and expressed as percentage) between Austropotamobius haplotypes of the mtDNA 16S rRNA

Haplotype A. italicus

A. pallipes

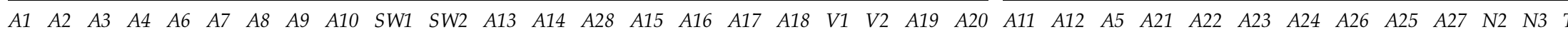

A

\begin{tabular}{ll}
\hline A1 & \\
A2 & 0.4 \\
A3 & 0.2
\end{tabular}

A3 $\quad 0.20 .2$

A4 $\quad 2.6 \quad 3.0 \quad 2.8$

A6 $\quad 1.9 \quad 1.4 \quad 1.6 \quad 4.0$

A7 $\quad \begin{array}{llllll}1.6 & 1.2 & 1.4 & 3.7 & 0.2\end{array}$

$\begin{array}{lllllllll}\text { A8 } & 1.2 & 1.2 & 0.9 & 3.2 & 0.7 & 0.5 & \\ \text { A9 } & & 2.3 & 2.3 & 2.1 & 2.1 & 3.7 & 3.5 & 3.0\end{array}$

$\begin{array}{llllllllll}\mathrm{A} 10 & 0.5 & 0.5 & 0.2 & 3.0 & 1.9 & 1.6 & 1.1 & 2.3\end{array}$

$\begin{array}{lllllllllll}\text { SW1 } & 0.5 & 0.9 & 0.7 & 3.0 & 2.3 & 2.1 & 1.6 & 2.8 & 0.9\end{array}$

$\begin{array}{lllllllllll}\text { SW2 } & 0.7 & 1.2 & 0.9 & 3.3 & 2.6 & 2.3 & 1.9 & 3.0 & 1.2 & 0.2\end{array}$

$\begin{array}{llllllllllll}\text { A13 } & 1.9 & 2.3 & 2.1 & 1.6 & 3.7 & 3.5 & 3.0 & 0.5 & 2.3 & 2.3 \\ \text { A14 } & & 2.1 & 2.1 & 1.9 & 1.9 & 3.5 & 3.3 & 2.9 & 0.2 & 2.1 & 2.6\end{array}$

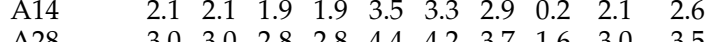

$\begin{array}{lllllllllll}\mathrm{A} 28 & 3.0 & 3.0 & 2.8 & 2.8 & 4.4 & 4.2 & 3.7 & 1.6 & 3.0 & 3.5 \\ \mathrm{~A} 15 & 2.6 & 3.0 & 2.8 & 0.5 & 4.0 & 3.7 & 3.3 & 2.1 & 3.0 & 3.0\end{array}$

$\begin{array}{lllllllllll}\text { A16 } & 2.8 & 2.8 & 2.6 & 0.7 & 3.7 & 3.5 & 3.0 & 1.9 & 2.8 & 3.3\end{array}$

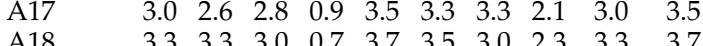

$\begin{array}{lllllllllll}\text { V1 } & 3.0 & 3.0 & 2.8 & 0.5 & 4.0 & 3.7 & 3.3 & 2.1 & 3.0 & 3.5\end{array}$

$\begin{array}{llllllllllll}\mathrm{V} 2 & 2.8 & 3.3 & 3.0 & 0.2 & 4.2 & 4.0 & 3.5 & 2.3 & 3.3 & 3.3\end{array}$

$\begin{array}{llllllllllll}\mathrm{A} 19 & 1.2 & 1.6 & 1.4 & 3.3 & 1.6 & 1.4 & 0.9 & 3.5 & 1.6 & 1.6\end{array}$

$\begin{array}{lllllllllll}\mathrm{A} 20 & 0.9 & 0.9 & 0.7 & 3.0 & 0.9 & 0.7 & 0.2 & 2.8 & 0.9 & 1.4 \\ \mathrm{~A} 11 & 4.4 & 4.0 & 4.2 & 4.2 & 4.9 & 4.7 & 4.7 & 4.2 & 4.4 & 4.9\end{array}$

$\begin{array}{lllllllllll}\mathrm{A} 12 & 4.7 & 4.2 & 4.4 & 4.4 & 5.1 & 4.9 & 4.9 & 4.4 & 4.7 & 5.1\end{array}$

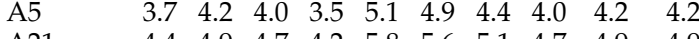

$\begin{array}{lllllllllll}\text { A21 } & 4.4 & 4.9 & 4.7 & 4.2 & 5.8 & 5.6 & 5.1 & 4.7 & 4.9 & 4.9 \\ \text { A22 } & 4.4 & 4.0 & 4.2 & 4.2 & 4.9 & 4.7 & 4.7 & 4.2 & 4.4 & 4.9\end{array}$

$\begin{array}{llllllllllll}\mathrm{A} 23 & 4.0 & 4.4 & 4.2 & 3.7 & 5.4 & 5.1 & 4.7 & 4.2 & 4.4 & 4.4\end{array}$

$\begin{array}{lllllllllll}\mathrm{A} 24 & 4.4 & 4.4 & 4.2 & 4.2 & 4.9 & 4.7 & 4.2 & 4.2 & 4.4 & 4.9\end{array}$

$\begin{array}{llllllllll}4.0 & 4.4 & 4.2 & 4.2 & 4.9 & 4.7 & 4.2 & 4.2 & 4.4 & 4.9 \\ 4.0 & 4.7 & 3.7 & 4.9 & 4.7 & 4.2 & 3.3 & 4.0 & 4.4\end{array}$

$\begin{array}{llllllllll}4.2 & 4.7 & 4.4 & 4.0 & 5.4 & 5.1 & 4.7 & 4.0 & 4.7 & 4.7\end{array}$

$\begin{array}{llllllllllll}\mathrm{A} 25 & 4.2 & 4.7 & 4.4 & 4.0 & 5.4 & 5.1 & 4.7 & 4.0 & 4.7 & 4.7 \\ \mathrm{~A} 27 & & 4.0 & 3.5 & 3.7 & 3.7 & 4.4 & 4.2 & 4.2 & 3.3 & 4.0 & 4.4\end{array}$

$\begin{array}{llllllllllll}\mathrm{A} 27 & 4.0 & 3.5 & 3.7 & 3.7 & 4.4 & 4.2 & 4.2 & 3.3 & 4.0 & 4.4 \\ \mathrm{~N} 2 & 4.0 & 4.4 & 4.2 & 3.7 & 5.4 & 5.1 & 4.7 & 3.7 & 4.4 & 4.4 \\ \mathrm{~N} 3 & 4.2 & 4.2 & 4.0 & 4.0 & 5.1 & 4.9 & 4.4 & 3.5 & 4.2 & 4.7\end{array}$

2.6

2.8 0.2

$\begin{array}{llll}3.3 & 1.6 & 1.9 & 2.4\end{array}$

$\begin{array}{lllll}3.5 & 1.9 & 1.6 & 2.1 & 0.2\end{array}$

$\begin{array}{llllll}3.7 & 2.1 & 0.9 & 2.3 & 0.4 & 0.2\end{array}$

$\begin{array}{lllllll}4.0 & 2.3 & 2.1 & 2.6 & 0.7 & 0.5 & 0.7\end{array}$

$\begin{array}{llllllll}3.7 & 2.1 & 1.9 & 2.3 & 0.5 & 0.2 & 0.5 & 0.2\end{array}$

$\begin{array}{lllllllll}3.5 & 1.9 & 2.1 & 2.6 & 0.2 & 0.5 & 0.7 & 0.5 & 0.2\end{array}$

$\begin{array}{llllllllll}1.9 & 3.0 & 3.3 & 4.2 & 3.3 & 3.5 & 3.7 & 3.5 & 3.7 & 3.5\end{array}$

$\begin{array}{lllllllllll}1.6 & 2.8 & 2.6 & 3.5 & 3.0 & 2.8 & 3.0 & 2.8 & 3.0 & 3.3 & 0.7\end{array}$

$\begin{array}{lllllllllllll}5.1 & 4.2 & 4.0 & 4.4 & 3.7 & 3.5 & 3.3 & 4.0 & 3.7 & 4.0 & 5.1 & 4.4\end{array}$

$\begin{array}{lllllllllllll}5.4 & 4.4 & 4.2 & 4.7 & 4.0 & 3.7 & 3.5 & 4.2 & 4.0 & 4.2 & 5.4 & 4.7 & 0.2\end{array}$

$\begin{array}{llllllllllllll}4.4 & 3.5 & 3.7 & 4.2 & 3.0 & 3.3 & 3.5 & 3.7 & 3.5 & 3.3 & 4.4 & 4.2 & 0.7 & 0.9\end{array}$

$\begin{array}{lllllllllllllll}5.1 & 4.2 & 4.4 & 4.9 & 3.7 & 4.0 & 4.2 & 4.4 & 4.2 & 4.0 & 5.1 & 4.9 & 1.4 & 1.2 & 0.7\end{array}$

$\begin{array}{llllllllllllllll}5.1 & 4.2 & 4.0 & 4.4 & 3.7 & 3.5 & 3.3 & 4.0 & 3.7 & 4.0 & 5.1 & 4.4 & 0.5 & 0.7 & 0.7 & 1.4\end{array}$

$\begin{array}{lllllllllllllllll}4.7 & 3.7 & 4.0 & 4.4 & 3.3 & 3.5 & 3.7 & 4.0 & 3.7 & 3.5 & 4.7 & 4.4 & 0.9 & 1.2 & 0.2 & 0.9 & 0.4\end{array}$

$\begin{array}{llllllllllllllllll}5.1 & 4.2 & 4.0 & 4.4 & 3.7 & 3.5 & 3.7 & 4.0 & 3.7 & 4.0 & 4.7 & 4.0 & 0.9 & 1.2 & 0.7 & 1.4 & 0.4 & 0.4\end{array}$

$\begin{array}{llllllllllllllllllll}4.7 & 3.3 & 3.0 & 4.0 & 3.3 & 3.0 & 3.3 & 3.5 & 3.5 & 3.3 & 3.5 & 4.7 & 4.0 & 0.9 & 1.2 & 0.7 & 0.9 & 0.9 & 0.9\end{array}$

$\begin{array}{llllllllllllllllllll}4.9 & 3.5 & 3.7 & 4.7 & 3.5 & 3.7 & 4.0 & 4.2 & 4.0 & 3.7 & 4.9 & 4.6 & 1.6 & 1.9 & 0.9 & 1.6 & 1.6 & 1.2 & 1.6 & 0.7\end{array}$

$\begin{array}{lllllllllllllllllllllll}4.7 & 3.3 & 3.0 & 4.0 & 3.3 & 3.0 & 2.8 & 3.5 & 3.3 & 3.5 & 4.7 & 3.9 & 0.9 & 1.2 & 1.2 & 1.9 & 0.9 & 1.4 & 1.4 & 0.4 & 1.2\end{array}$

$\begin{array}{lllllllllllllllllllllllllllllllllll}4.0 & 4.4 & 4.2 & 3.7 & 5.4 & 5.1 & 4.7 & 3.7 & 4.4 & 4.4 & 4.7 & 3.2 & 3.5 & 4.4 & 3.3 & 3.5 & 3.7 & 4.0 & 3.7 & 3.5 & 4.7 & 4.4 & 1.4 & 1.2 & 0.7 & 0.9 & 1.4 & 0.9 & 1.4 & 0.5 & 0.7 & 0.9\end{array}$

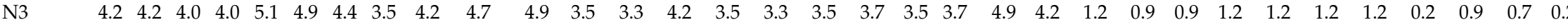

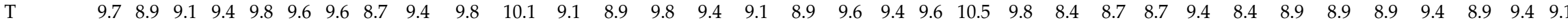

$\mathrm{T}=$ Austropotamobius torrentium. Insertions/deletions are included. 


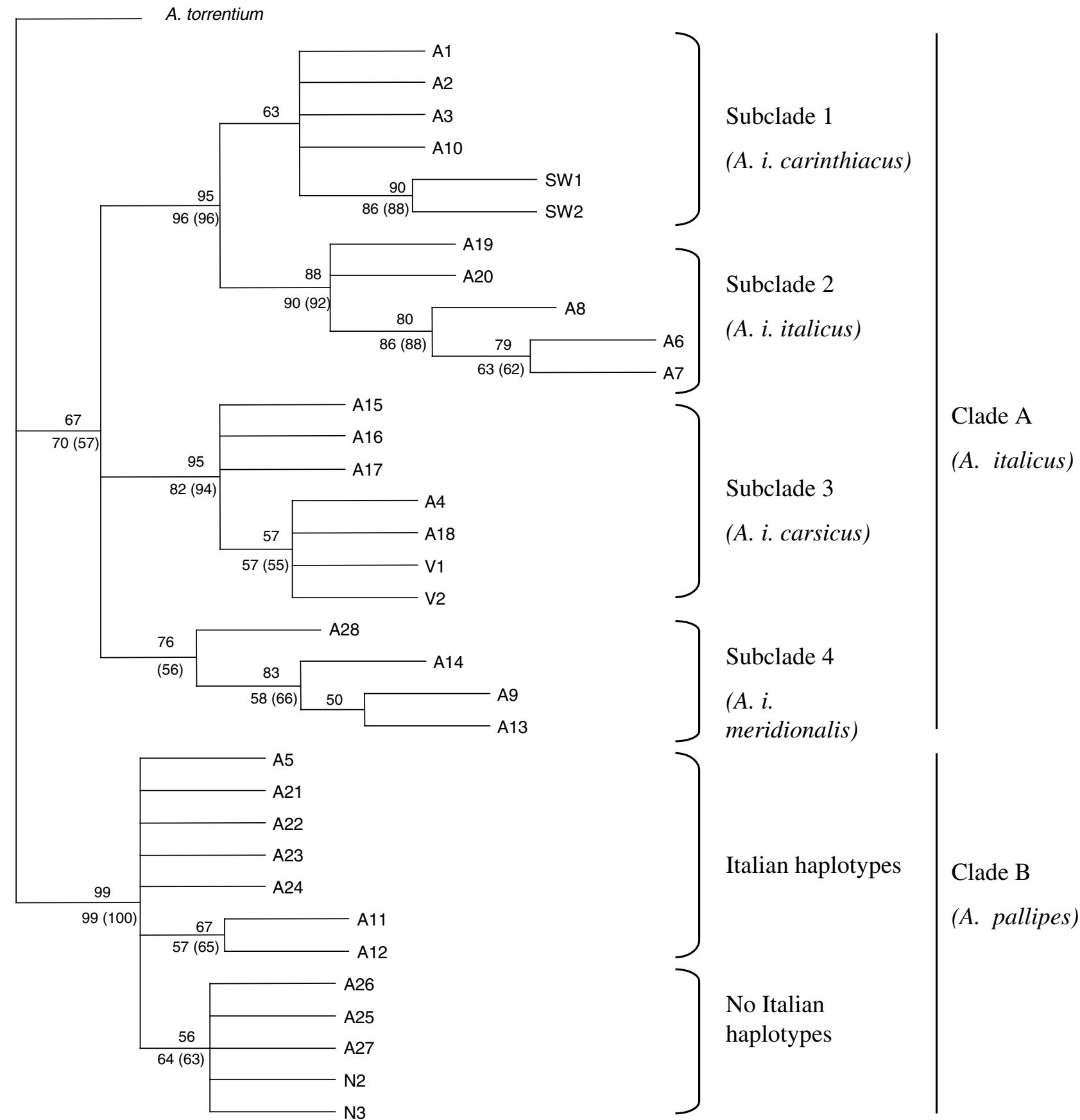

Figure $2 \mathrm{NJ}$ tree inferred from the analysis of $486 \mathrm{bp}$ of the mtDNA 16S rRNA gene of Austropotamobius spp. Bootstrap values are given above nodes (2000 replications; only confidence values higher than 50\% are shown in the tree). Numbers below the nodes are the bootstrap values for ML tree (250 replications) and MP consensus tree (in parentheses, 1000 replications). The haplotype designations correspond to those reported in Table 1. Clade A corresponds to A. italicus and clade B to A. pallipes. The subclades designed 1-4 indicate the $A$. italicus subspecies.

haplotypes; 7.2-8.6 between A. torrentium and A. italicus haplotypes.

Within clade A, four subclades could be defined, corresponding to well-defined geographic zones (Figures 2 and 3): subclade 1 corresponds to haplotypes from Central and North-Western Italy; subclade 2 includes haplotypes corresponding to the Tuscan-Emilian Apennine; subclade 3 groups haplotypes from North-Eastern Italy and haplotypes from Orobie Alps; and subclade 4 includes haplotypes from Latium, Abruzzi, South Italy, and Slovenia sites.
In a population (no. 9 in Table 1), we recorded the presence of haplotypes corresponding to the two different Austropotamobius species. The genetic distance within and between subclades is reported in Table 4 .

\section{Discussion}

\section{Taxonomic inferences}

The phylogenetic reconstruction reported in Figure 2 reveals complex evolutionary relationships among 
Italian populations of the white-clawed crayfish, suggesting the occurrence of distinct evolutionary units. First, our results confirm the presence of both $A$. pallipes and $A$. italicus in the Italian peninsula and, in agreement with Nascetti et al (1997) and Santucci et al (1997), define the distribution zone of the former as restricted to the North-

Table 3 16S rRNA variable sites discriminating the two Austropotamobius species

\begin{tabular}{lcccccc}
\hline & \multicolumn{6}{c}{ Variable sites } \\
\cline { 2 - 7 } & 29 & 162 & 173 & 194 & 238 & 267 \\
\hline A. pallipes & G & T & T & G & C & G \\
A. italicus & A & T-C & C & A & T & A \\
\hline
\end{tabular}

Western Italy, and of the latter as ranging across all the Italian peninsula (Figure 3). The two species overlap in the Ligurian Apennine, where we also found individuals of the two species inhabiting the same watercourse. It seems likely that this mixed population is the result of a natural secondary contact between the two species during their spreading from different refugia after glacial events (see below). Alternatively, it may be the effect of human translocation, considering the high anthropic impact on the distribution of the white-clawed crayfish all over Europe (Souty-Grosset et al, 1997; Grandjean et al, 2000; Largiadèr et al, 2000). We recorded no data on events of hybridization between the two species, but this phenomenon has been excluded by other, more extensive, genetic studies (Nascetti et al, 1997; Santucci et al, 1997). However, a morphological study (Froglia, 1978)

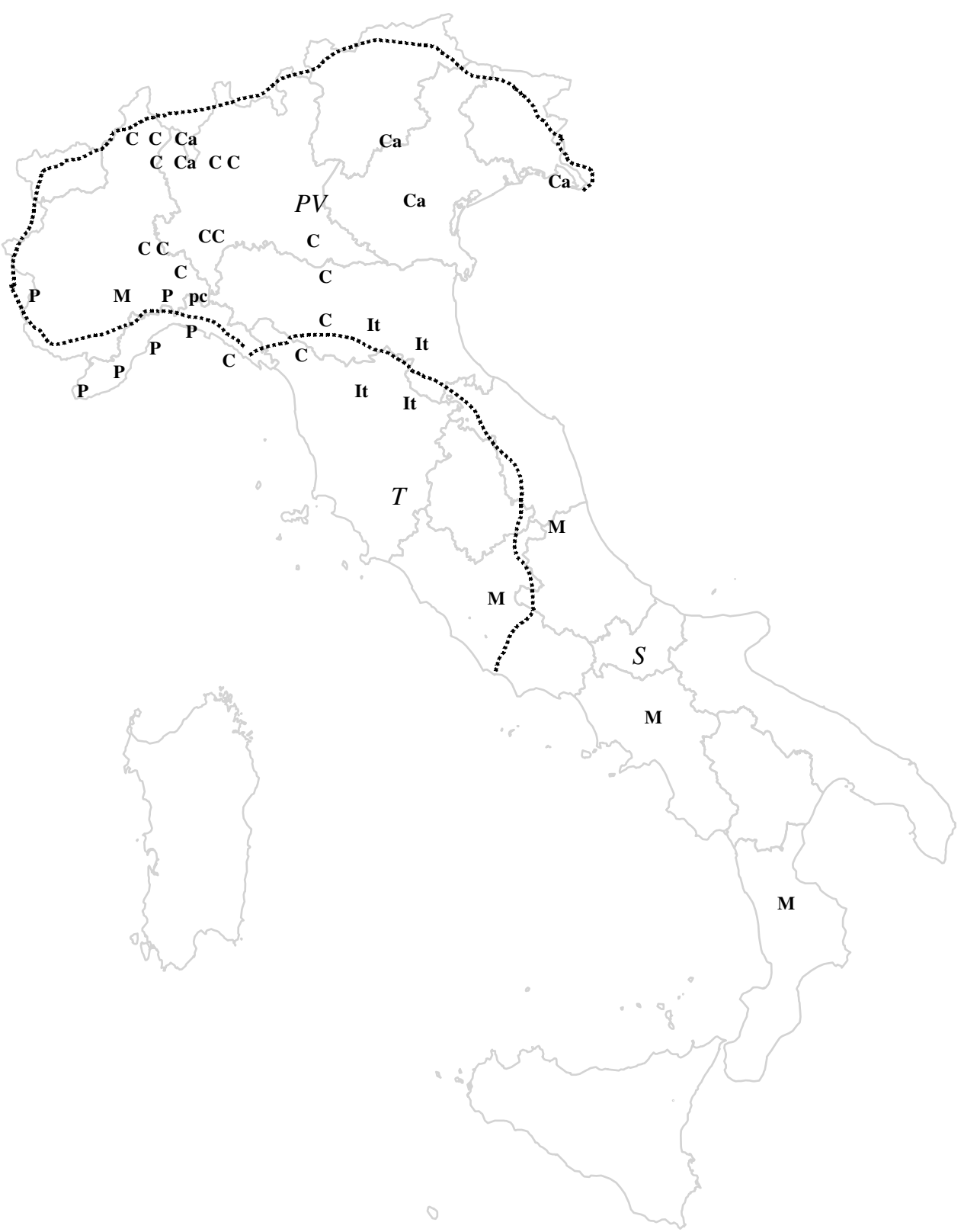

Figure 3 Geographic distribution of $A$. pallipes and A. italicus in Italy. Symbols on the map indicate: $\mathrm{C}=$ A. i. carinthiacus; It $=$ A. i. italicus, $\mathrm{M}=$ A. $i$. meridionalis; $\mathrm{Ca}=A$. $i$ carsicus; $\mathrm{P}=A$. pallipes; $\mathrm{pc}=A$. pallipes and $A$. $i$. carinthiacus mixed population; $\mathrm{PV}=\mathrm{Padan}-\mathrm{Venetian}$ ichthyogeographic district; $\mathrm{T}=$ Tuscan-Latium district; $\mathrm{S}=$ Southern Italy district. 
Table 4 Mean sequence divergences (calculated as $p$-distance=number of substitutions/total number of nucleotides examined and expressed as percentage) \pm standard errors within and among Austropotamobius subspecies and species

\begin{tabular}{|c|c|c|c|c|c|}
\hline & Clade 1 (A. i. carinthiacus) & Clade 2 (A. i. italicus) & Clade 3 (A. i. carsicus) & Clade 4 (A. i. meridionalis) & Clade B (A. pallipes) \\
\hline Clade 1 & $0.41 \pm 0.21$ & & & & \\
\hline Clade 2 & $1.10 \pm 0.43$ & $0.24 \pm 0.16$ & & & \\
\hline Clade 3 & $3.01 \pm 0.79$ & $3.08 \pm 0.84$ & $0.27 \pm 0.17$ & & \\
\hline Clade 4 & $2.45 \pm 0.66$ & $3.07 \pm 0.77$ & $2.01 \pm 0.59$ & $0.84 \pm 0.30$ & \\
\hline Clade B & $3.81 \pm 0.91$ & $3.91 \pm 0.96$ & $3.06 \pm 0.84$ & $3.36 \pm 0.84$ & $0.36 \pm 0.15$ \\
\hline
\end{tabular}

Gaps are excluded.

revealed the existence in the Ligurian Apennine (ie the overlapping zone between the two species) of crayfish having intermediate features between $A$. pallipes and $A$. italicus. In the future, more extensive genetic, morphological, and behavioural studies will be directed to clarify the potential of hybridization between the two species.

The current taxonomic position of the A. pallipes complex, based on the mtDNA $16 S$ gene (Grandjean et al, 2000, 2002a,b), distinguishes three A. italicus subspecies. Thank to the inclusion of reference sequences for all the $A$. italicus subspecies (Table 1), our results clearly show the presence of all of them in Italy (Figures 2 and 3): A. i. italicus in the Tuscan-Emilian Apennine (ie Central Italy); A. i. carsicus in the North-Eastern Italy; and A. $i$. carinthiacus in the Central and North-Western Italy. We found a fourth separate subclade within $A$. italicus (named 4 in Figure 2), corresponding to haplotypes from Latium, Abruzzi, and Southern Italy sites and including also the Slovenian haplotype. This clade does not correspond to any of the species and subspecies described by previous systematic revisions (Bott, 1950, 1972; Karaman, 1962; Brodsky, 1983; Starobogatov, 1995; Grandjean et al, 2000, 2002a, b). Since the pairwise genetic separation between this and the other subclades is of the same magnitude with respect to each pairwise subclade comparison, we can consider it a further $A$. italicus subspecies, following the taxonomic criterion used by Grandjean et al (2000, 2002a, b). Owing to its geographic distribution in Italy (Figure 3), we name this new subspecies A. i. meridionalis. The only two works (Nascetti et al, 1997; Santucci et al, 1997) that included in their collections samples from Southern Italy underlined their genetic separation from the rest of Italy by the analysis of allozyme diversity, but these results were not discussed within the frame of systematics.

The level of genetic variation within A. pallipes, on the one hand, is consistently lower with respect to that recorded for $A$. italicus ( $p$-distance: $0.36 \pm 0.15$ versus $2.0 \pm 0.04 \%$ ) and, on the other, is comparable to the variation found within each $A$. italicus subspecies. This result could be explained by a more restricted distribution range for $A$. pallipes, limited to North Apennine, as compared to A. italicus. However, A. pallipes haplotypes from Italian sites (named A5, A11, A12; A21, A22; A23 and A24), even if not resolved in the evolutionary trees (Figure 2), are separated from the haplotypes from France, Ireland, and Switzerland, which form a monophyletic group within the species $A$. pallipes. This result may confirm the presence of two $A$. pallipes subspecies, as proposed by Brodsky (1983), A. p. pallipes in France and the British Isle, and $A$. p. bispinosus confined to Italy. Anyway, independently from the systematic implication, this result can be viewed as an additional example of the role played by mountain chains in the separation events, as reported for other freshwater species such as the cyprinid fish vairone (Leuciscus souffia multicellus) (Salzburger et al, 2003).

\section{Biogeographical implications}

Our results clearly show the occurrence in Italy of two species and four subspecies of the genus Austropotamobius and shed light on their biogeography (Figures 2 and 3 ). The scenario we depict here seems to be the result of events that occurred from the Pleistocene until recent historical times. In fact, it has been extensively claimed (reviewed in Hewitt, 2000) that strong climatic oscillations occurring during the past $3 \mathrm{Myr}$ (with the series of major ice ages) both increased speciation phenomena and divergence of present lineages, and influenced the distribution of many species in Europe, masking the effect of older events. In particular, an explanation for both the high inter- and intraspecific genetic variability of Italian crayfish and their present geographic distribution might be that the four $A$. italicus lineages survived in separate glacial refugia, in accordance with the assumption of multiple refugia by Banarescu (1992). These refugia could be located in Southern Italy, Central Italy, and the Balkans that, together with Spain, Greece, and Turkey, were typical ice-age refugia for many freshwater species during the Pleistocene (Pretzmann, 1987; Hewitt, 1999, 2000), and consequently became the depository of exclusive genetic entities during the post-glacial expansion events (for a review, see Hewitt, 2000).

Bianco (1995) distinguished three main ichthyogeographic districts in Italy (Figure 3): the Padan-Venetian (PV) district, which includes the rivers flowing into the upper and middle Adriatic Sea; the Tuscan-Latium (TL) district, ranging from the rivers Serchio and Arno, in Tuscany, up to the Tiber, flowing into in the Tyrrenian Sea; and the Southern Italy district (S), including all the Southern rivers flowing into both the Eastern and Western Italian coasts. As shown in Figure 3, the distribution of $A$. italicus lineages seems inconsistent with the Italian ichthyogeographic districts (Bianco, 1993, 1995) and with the Apennine chain that acts as a barrier to the dispersion of most fish species (Bianco, 1993; Salzburger et al, 2003; Stefani et al, 2004). As shown in Figure 3, with the exception of $A$. i. carsicus entirely located within the PV district, the distribution ranges of all the other Austropotamobius lineages include two distinct ichthyogeographic districts. On the one hand, this may be the effect of human translocation, which has affected the distribution of many commercially 
important and comestible freshwater species in the recent past in Italy as well as all over Europe (Bianco, 1987, 1993, 1995; Balon, 1995). On the other hand, the distribution of $A$. italicus may be related to plate-tectonic events at a local level along the Apennine ridge, which caused the capture of rivers between their headwaters (Cattauto et al, 1988). Due to these phenomena, coldwater species (such as the European crayfish inhabiting mountain streams) might have crossed high mountains and occupied adjacent hydrographic districts (Bianco, 1993; Stefani et al, in press).

A. i. carinthiacus (Central and North-Western Italy) and A. i. italicus (Central Apennine) are the most closely genetically related subspecies (Table 3 ). This result suggests that these two lineages, presently inhabiting distinct basins (the Po river and the Central Apennine river system, respectively), originated from the same glacial refuge and then became isolated due to the interruption of connections among the two basins. The high genetic similarity among these two subspecies can also explain why Nascetti et al (1997) and Santucci et al (1997) found three (and not four) evolutionary lineages within $A$. italicus. In fact, using a less variable marker (ie allozymes), these authors were unable to distinguish two separate lineages in the North-Central Apennines, where, on the contrary, we found $A$. i. carinthiacus and A. i. italicus.

A. i. carsicus and A. i. carinthiacus overlap in the Orobie Alps, as shown in the populations of the Lambro river (sites 16-19: Table 1) (Figure 3). The Orobie Alps could represent the confluence point of these two evolutionary lineages when they expanded their range from separate glacial refugia. On the other hand, we cannot exclude the overlapping between these two subspecies being the effect of an artificial translocation. Since the beginning of 1900s, this area has been affected by the effects of a high level of urbanisation and has seen a decrease in the number of crayfish populations (Mazzarelli, 1903), followed by human re-introductions.

A. i. meridionalis is located in Central-Southern Italy and in Slovenia: the haplotype from Slovenia (Table 1) in its original source (Grandjean et al, 2000) was reported to correspond to A. i. carsicus (typical of Balkans and NorthEastern Italy). This can be interpreted as an artifact due to the impossibility of Grandjean et al (2000) to correctly resolve the taxonomic position of this haplotype without the inclusion of other locations in the distribution range of this new subspecies. However, the presence of the same $A$. italicus subspecies in South Italy and Slovenia is rather unexpected. In fact, Slovenia belongs to the northern ichthyologic district (PV: Bianco, 1990) and is considered an exchange area between PV and the Danubian ichthyofaunas (Durand et al, 1999; Tsigenopoulos and Berrebi, 2000). It was thus more logical to find $A$. i. carsicus there. A possible explanation could be that, during the Pleistocene, crayfish that took refuge in Southern Italy have colonized Slovenia due to the lowering sea level and the consequent confluence of some Adriatic rivers (Bianco, 1995). Such a scenario has also been proposed to explain the Italian and Balkans distribution of the bullhead Cottus gobio (Bianco, 1993), therefore enforcing our hypothesis. But, again, this result might be a consequence of human translocation. A more extensive genetic study on Slovenian populations will be necessary to clarify this point.
Our genetic results on $A$. pallipes showing two distinct lineages (one in France and one in Italy) suggest two $A$. pallipes refugia in the Alps, one on the French (already suggested by Grandjean et al, 1998) and the other on the Italian side.

\section{Conservation issues}

This study revealed that Italy, where two Austropotamobius species and four $A$. italicus subspecies are present, is the depository of an elevated haplotype variability, never described before throughout Western Europe. Therefore, this country may be considered a 'hot-spot' (sensu IUCN, Baillie and Groombridge, 1996) for the genetic diversity of the European native crayfish Austropotamobius.

The occurrence of five taxonomic units of this threatened crayfish in Italy clearly suggests that any conservation program and re-introduction plan should refer to the geographic distribution of each unit to ensure the preservation of independent genetic pools. Moreover, in the light of the recorded human effect on the distribution of the different evolutionary units, it is desirable that any population would be genetically screened before any management action. Particular attention is needed in the overlapping areas between species and subspecies where genetically distinct units can occur even within the same water body. To discriminate among Austropotamobius species, a rapid and economic screening may be based on the analysis of the six variable sites (Table 2); in the case of $A$. italicus, an additional genetic analysis will be necessary to establish the subspecies.

In Piedmont (North-Western Italy), we found a population (corresponding to no. 6 in Table 1 and Figure 1) with one Southern Italy haplotype (haplotype A13), which resulted from human translocations and not from natural migration (because of the distance between the distribution ranges of the two subspecies). This record is somewhat alarming since it shows that in the recent past uncontrolled re-introduction initiatives have been performed without any concern of the evolutionary history of the species. These attempts can be unsuccessful and economically wasteful due to the introduction of individuals that are not genetically adapted to that environment. Besides, they can compromise the natural genetic pool of the species in the area of introduction, with the loss of genetic identity of local populations as a result of competition and hybridization between local and introduced individuals.

Future studies will be directed to identify within each lineage demographically and genetically independent populations that may be designated as separate management units (MUs; Moritz, 1994). Such knowledge will help define conservation priorities by identifying those populations with the highest genetic variability and therefore with the highest resistance to environmental changes (Soulé and Mills, 1992; Primack, 2000). Management efforts and economic resources should be concentrated on these populations.

\section{Acknowledgements}

Funding was provided by the Italian Ministry of Agriculture and Forestry (MIPAF, 'Sesto piano triennale per la pesca e l'acquacoltura', no. 119) to Francesca Gherardi and by EU (Life-Nature 2000 Project) to 
Giuseppe Crosa. We express our thanks, for collection of samples, to: Tamara Jug; Almo Farina; Massimo Scalici; Fabrizio Stefani; Paolo Galli; Pietro Angelo Nardi; Edoardo Razzetti; Alessandro Negri; Bruno Maiolini; Nicola Bressi; Guido Crudele; Guardie Provinciali of La Spezia, Salerno and Cosenza (Italy); Angelo Morisi; Federica Cenni; Paolo Bazzoni. We also thank an anonymous referee for his useful comments to the manuscript.

\section{References}

Baillie J, Groombridge B (1996). Red List of Threatened Animals. IUNC: Gland, Switzerland.

Balon EK (1995). Origin and domestication of the wild carp, Cyprinus carpio: from Roman gourmets to the swimming flowers. Aquaculture 129: 3-48.

Banarescu PM (1992). Principaux traits de la zoogéographie des eaux douces. C R Soc Biog 67: 133-154.

Bianco PG (1987). L'inquadramento zoogeografico dei pesci d'acqua dolce d'Italia e problemi determinati dalle falsificazioni faunistiche. Atti II Conv. AIIAD: Torimo, Italy, pp 41-65.

Bianco PG (1990). Potential role of the paleohistory of the Mediterranean and Paratethys basins on the early dispersal of Euro-Mediterranean freshwater fishes. Icht Expl Fresh 1: 167-184.

Bianco PG (1993). L'ittiofauna continentale dell'Appennino umbro-marchigiano, barriera semipermeabile allo scambio di componenti primarie tra gli opposti versanti dell'Italia centrale. Biogeographia 12: 427-485.

Bianco PG (1995). Mediterranean endemic freshwater fishes in Italy. Biol Cons 72: 159-170.

Bott R (1950). Die Flusskrebse europas (Decapoda; Astacidae). Proc Senck Nat Soc 483: 1-36.

Bott R (1972). Besiedlungsgeschichte und Systematik der Astaciden West-Europas unter besonderer Berücksichtigung der Schweiz. Rev Suisse Zool 79: 387-408.

Brodsky SY (1983). On the systematic of palearctic crayfishes (Crustacea, Decapoda). Freshwater Crayfish 5: 464-470.

Cabot EL, Beckenbach AT (1989). Simultaneous editing of multiple nucleic acid and protein sequences with ESEE. Comp Appl Bios 5: 233-234.

Cattauto C, Cencetti C, Gregori L (1988). Lo studio dei corsi d'acqua minori dell'Italia Appenninica come mezzo di indagine sulla tettonica del Plio/Pleistocene. Boll Mus St Nat Lunigiana 6-7: 7-10.

Clary DO, Wolstenholme DR (1985). The mitochondrial DNA molecule of Drosophila yakuba: nucleotide sequence, gene organization, and genetic code. J Mol Evol 22: 252-271.

Crandall KA (1996). Genetic variation within and among crayfish species. Freshwater Crayfish 11: 135-145.

Crandall KA, Fitzpatrick Jr JF (1996). Crayfish molecular systematics: using a combination of procedures to estimate phylogeny. Syst Biol 45: 1-26.

Durand JD, Persant H, Bouvet Y (1999). Phylogeography and postglacial dispersion of the chub (Leuciscus cephalus) in Europe. Mol Ecol 8: 989-997.

Erwin TL (1991). An evolutionary basis for conservation strategies. Science 253: 750-752.

Felsenstein J (1985). Confidence limits on phylogenies: an approach using the bootstrap. Evolution 39: 783-791.

Frankham R, Ballou JD, Briscoe DA (2002). Introduction to Conservation Genetics. Cambridge University Press: Cambridge.

Froglia C (1978). Decapodi (Crustacea Decapoda). In: Ruffo S (ed) Guide per il riconoscimento delle specie animali delle acque interne italiane, $A Q / 1 / 9 / 4$. Consiglio Nazionale delle Ricerche: Verona, Italy.

Grandjean F, Bouchon D, Souty-Grosset C (2002a). Systematic of the European endangered crayfish species Austropotamobius pallipes (Decapoda: Astacidae) with a re-examination of the status of Austropotamobius berndhauseri. I Crust Biol 22: 677-681.

Grandjean F, Frelon-Raimond M, Souty-Grosset C (2002b) Compilation of molecular data for the phylogeny of the genus Austropotamobius: one species or several? Bull Fr PêchePiscic 367: 671-680.

Grandjean F, Gouin N, Frelon M, Souty-Grosset C (1998). Genetic and morphological systematic studies on the crayfish Austropotambius pallipes. J Crust Biol 18: 549-555.

Grandjean F, Harris DJ, Souty-Grosset C, Crandall KA (2000). Systematic of the European endangered crayfish species Austropotamobius pallipes (Decapoda: Astacidae). J Crust Biol 20: 522-529.

Hasegawa M, Kishino K, Yano T (1985). Dating the human-ape splitting by a molecular clock of mitochondrial DNA. J Mol Evol 22: 160-174.

Hewitt GM (1999). Post-glacial recolonization of European biota. Biol J Linn Soc 68: 87-112.

Hewitt GM (2000). The genetic legacy of the Quaternary ice ages. Nature 405: 907-913.

Karaman MS (1962). Ein Beidrag zur Systematic der Astacidae (Decapoda). Crustaceana 3: 173-191.

Kocher TD, Thomas WK, Meyer A, Edwards SV, Paabo S, Villablancha FX (1989). Dynamics of mtDNA evolution in animals: amplification and sequencing with conserved primers. Proc Natl Acad Sci USA 86: 6196-6200.

Largiadèr CR, Herger F, Lörtscher M, Choll A (2000). Assessment of natural and artificial propagation of the whiteclawed crayfish (Austropotamobius pallipes species complex) in the Alpine region with nuclear and mitochondrial markers. Mol Ecol 9: 25-37.

Lörtscher M, Stucki TP, Clalüna M, Scholl A (1997). Phylogeographic structure of Austropotamobius pallipes populations in Switzerland. Bull Fr PêchePiscic 347: 649-661.

Mazzarelli G (1903). La pesca dei gamberi nella provincia di Milano. Commissione per la pesca commerciale di questi crostacei per la Provincia di Milano: Milano, Italy.

Moritz C (1994). Defining 'evolutionary significant units' for conservation. Trends Ecol Evol 9: 373-375.

Nascetti G, Andreani P, Santucci F, Iaconelli M, Bullini L (1997). Struttura genetica di popolazioni italiane di gambero di fiume (Austropotamobius pallipes) e strategie per la sua conservazione. S It E Atti 18: 205-208.

Palumbi S, Martin A, Romano S (1991). The Simple Fool's Guide to PCR. Department of Zoology and Kewalo Marine Laboratory, University of Hawaii: Honolulu, Hawaii.

Posada D, Crandall KA (1998). MODELTEST: testing the model of DNA substitution. Bioinformatics 14: 817-818.

Pretzmann G (1987). A contribution to a historic analysis of Mediterranean freshwater decapods chorology. Invest Pesq 51: 7-25.

Primack RB (2000). Conservation at the population and species level. In: Primack RB (ed) A Primer of Conservation Biology, 2nd edn. Sinauer Associates: Sunderland, MA, pp 121-182.

Salzburger W, Brandstätter A, Gilles A, Parson W, Hempel M, Sturmbauer C et al (2003). Phylogeography of the vairone (Leuciscus souffia, Risso 1826) in Central Europe. Mol Ecol 12: 2371-2386.

Santucci F, Iaconelli M, Andreani P (1997). Allozyme diversity of European freshwater crayfish of the genus Austropotam bius. Bull Fr Pêche Piscic 347: 663-676.

Soulé ME, Mills LS (1992). Conservation genetics and conservation biology: a troubled marriage. In: Saundlund OT, Hindar K, Brown AHD (eds) Conservation of Biodiversity for Sustainable Development. Scandinavian University Press: Oslo, Scandinavia, pp 55-69.

Souty-Grosset C, Grandjean F, Raimond R, Frelon M, Debenest C, Bramard M (1997). Conservation genetics of the whiteclawed crayfish Austropotambius pallipes: usefulness of the mitochondrial DNA marker. Bull Fr Pêche Piscic 347: 677-692. 
Starobogatov YI (1995). Taxonomy and geographical distribution of crayfishes of Asia and East Europe (Crustacea, Decapoda, Astacoidea). Arthropoda Selecta 4: 3-25.

Stefani F, Galli P, Crosa G, Zaccara S, Calamari D (2004). Alpine and Apennine barriers determining the differentiation of the rudd (Scardinius erythrophthalmus L.) in Italian peninsula. Ecol Freshwater Fish 13: 168-175.

Stefani F, Galli P, Zaccara S, Crosa G. Genetic variability and phylogeography of the cyprinid T. multicellus Bonaporte,
1873 within the Italian peninsula as revealed by mitochondrial DNA. I Zool Syst Evol Res (in press).

Swofford DL (1998). PAUP* - Phylogenetic Analysis Using Parsimony ( ${ }^{*}$ and Other Methods) Version 4. Sinauer Associates: Sunderland, MA.

Tsigenopoulos CS, Berrebi P (2000). Molecular phylogeny of north Mediterranean barbs (genus Barbus: Cyprinidae) inferred from cytochrome $b$ sequences: biogeographic and systematic implications. Mol Phyl Evol 14: 165-179. 